\title{
Perceptual grouping and attention: Not all groupings are equal
}

\author{
RUTH KIMCHI and IRENE RAZPURKER-APFELD \\ University of Haifa, Haifa, Israel
}

\begin{abstract}
We examined grouping under inattention using Driver, Davis, Russell, Turatto, \& Freeman's (2001) method. On each trial, two successive displays were briefly presented, each comprising a central target square surrounded by elements. The task was to judge whether the two targets were the same or different. The organization of the background elements stayed the same or changed, independently of the targets. In different conditions, background elements grouped into columns/rows by color similarity, a shape (a triangle/arrow, a square/cross, or a vertical/horizontal line) by color similarity, and a shape with no other elements in the background. We measured the influence of the background on the target same-different judgments. The results imply that background elements grouped into columns/rows by color similarity and into a shape when no segregation from other elements was involved and the shape was relatively "good." In contrast, no background grouping was observed when resolving figure-ground relations for segregated units was required, as in grouping into a shape by color similarity. These results suggest that grouping is a multiplicity of processes that vary in their attentional demands. Regardless of attentional demands, the products of grouping are not available to awareness without attention.
\end{abstract}

Traditional theories of perception have assumed that perceptual grouping is a unitary process that occurs at an early, preattentive stage of processing, providing the perceptual units to which attention is allocated for later, more elaborated processing (e.g., Marr, 1982; Neisser, 1967; Treisman, 1982).

Empirical findings concerning the role of attention in grouping are rather confusing. Some findings obtained in texture segregation and visual search studies have led researchers to claim that grouping is accomplished by preattentive mechanisms (Julesz, 1984; Treisman, 1982). Other visual search studies have shown that some grouping involves focused attention (Enns \& Kingstone, 1995; Kimchi, 1998; Trick \& Enns, 1997). Ben-Av, Sagi, and Braun (1992), using the dual-task paradigm, and Mack, Tang, Tuma, Kahn, and Rock (1992), using the inattention paradigm, argued that no grouping can take place without attention. Yet results from studies that provided on-line measures of unattended processing suggest that some grouping can occur without attention (Driver, Russell, \& Howlett, unpublished data, cited in Driver, Davis, Russell, Turatto, \& Freeman, 2001; Moore \& Egeth, 1997).

This apparent confusion is partly due to different tasks, measures, and attention conditions. For example, visual search involves distributed attention and intention to

This research was conducted at the Institute of Information Processing and Decision Making, University of Haifa, and was partly based on I.R.-A.'s dissertation under the supervision of R.K. We thank Yafa Lev for programming the experiments, Anat Hershkovitz for help in data collection, and Daniel Algom and three anonymous reviewers for useful comments on an earlier version of this article. Correspondence should be addressed to R. Kimchi, Department of Psychology, University of Haifa, Haifa 31905, Israel (e-mail: rkimchi@research.haifa.ac.il). search, and thus does not really examine processing under inattention. Also, poor knowledge about unattended grouping, manifested in retrospective surprise questions (Mack et al., 1992), may reflect poor explicit memory rather than absence of processing of the unattended stimulus.

Another reason that may have contributed to the confusion is that different studies have examined different grouping processes with varying attentional demands. Several investigators have proposed that grouping is not a unitary process, but rather involves two distinct processes: (1) a process of unit formation or clustering that determines which elements belong together and are segregated from other elements, and (2) a process of shape formation or configuring that determines how the grouped elements appear as a whole based on the interrelations of the elements (Koffka, 1935; Rock, 1986; Trick \& Enns, 1997). Trick and Enns (1997) suggested that shape formation requires attention, whereas clustering does not. There are, however, some hints for a continuum, rather than a dichotomy, of attentional demands. Han and Humphreys (1999) presented subjects with local triangles embedded in a background of local crosses and grouped into columns/ rows or into a global triangle/arrow. Increasing the contrast of the local crosses (to match that of the local triangles) slowed identification of the global triangle/arrow, but not identification of the columns/rows, suggesting that the former involved more attentional effort than the latter. Han, Humphreys, and Chen (1999) found that responses to global shapes composed of local elements were slower when the elements were embedded in a background of other elements than when presented without background elements, suggesting that the identification of the global shape was more demanding when segmenta- 
tion from background elements was involved. Recently, Behrmann and Kimchi (2003) studied perceptual organization in two agnosic patients. Their patients had no problem grouping elements into columns/rows by proximity or by luminance similarity, but they exhibited different degrees of difficulty grouping elements into a global shape.

In this article, we report new evidence demonstrating that different groupings vary in their attentional demands and delineate some conditions that affect the attentional demands of grouping.

We used Driver et al.'s (2001; Russell, Howlett, \& Driver, 2000) paradigm to study grouping under inattention. This method provides on-line measures of unattended processing and enables manipulation of unattended grouping. On each trial, observers are presented with two successive displays that appear briefly. Each display includes a central target (a square made up of random black and white pixels) surrounded by background elements. The observer's task is to judge whether the two successive target squares are the same or different. When they are different, only a single pixel changes, rendering the task sufficiently demanding to absorb attention. The organization of the background elements stays the same or changes across successive displays, independently of whether the successive targets are the same or different. The influence of the background on the speed and accuracy of the same-different judgments is measured. If background grouping occurs without attention, targetsame judgments would be faster and/or more accurate when the background organization stays the same than when it changes, and target-different judgments would be faster and/or more accurate when the background organization changes than when it stays the same. After the last experimental trial, subjects are asked surprise questions about the immediately preceding background.

\section{EXPERIMENT 1}

We employed four background conditions. One condition was grouping elements into columns/rows by color similarity (Figure 1A). The second condition was grouping elements into a triangle/arrow by color similarity (Figure 1B). Both groupings involved the same factor of color similarity. Shape formation, however, may have been less demanding in the former, which required determination of the vertical or horizontal orientation of the groups, than in the latter, which required the formation of a distinctive shape (Rock, 1986). The third condition also required grouping elements into a triangle/ arrow (Figure 1C), but because several Gestalt factors including proximity and similarity (of color, luminance, and shape) supported the grouping, and no segregation from other elements was involved, it may have been less demanding than grouping into a triangle/arrow by color similarity. In the fourth condition, the background consisted of a connected triangle/arrow (Figure 1D). No grouping of elements was involved in this control condi- tion. The colors of the elements always changed between the two successive displays to disentangle a change in organization from a change in color per se.

If the process of shape formation cannot take place without attention, an effect of the background on subjects' performance would be expected only for the control condition but not for any of the grouping conditions. If, however, the attentional demands of grouping vary as a function of the complexity of shape formation (i.e., columns/rows vs. triangle/arrow), or as a function of the ease of segmenting the shape from the background (i.e., triangle/arrow with vs. without other background elements), a differential effect of the background would be expected for the different grouping conditions.

\section{Method}

Subjects. Fifty-six students at the University of Haifa served as subjects (14 in each of the four background conditions). All had normal or corrected-to-normal vision and normal color vision.

Stimuli. Each display consisted of a central target square surrounded by background elements. Viewing distance was $60 \mathrm{~cm}$. The central target square was made of 12 black and 13 white small solid squares, $.19^{\circ}$ each, randomly located in a $5 \times 5$ matrix subtending $.95^{\circ} \times .95^{\circ}$. The central target square stayed the same between two successive displays in half of the trials and changed in the other half. The change was made by switching the location of one small black square with that of a white one.

The elements in the grouping conditions were equidistant, equiluminant colored (blue, yellow, red, or green) solid circles, each $.48^{\circ}$ in diameter, located in a $9.1^{\circ} \times 9.1^{\circ}$ square matrix. The colors of the circles always changed between successive displays (blue and yellow in the first, and red and green in the second, or vice versa). The organization of the background circles either remained the same between successive displays or changed. Examples of the stimulus displays are presented in Figure 1.

In the columns/rows by color similarity condition (Figure 1A), 36 circles, 18 of one color and 18 of a different color, were grouped into columns or rows by color similarity. The distance between every two circles was $1.34^{\circ}$ and the distance of the most central circles from the target square was $.3^{\circ}$. In the triangle/arrow by color similarity condition (Figure 1B), 48 circles, 12 of one color and 36 of a different color, were grouped by color similarity into an isosceles triangle or an arrow, with each of its right angle sides subtending $6.24^{\circ}$. The distance between every two circles was $0.96^{\circ}$, and the distance of the most central circles from the square target was $.7^{\circ}$. In the triangle/arrow condition (Figure 1C), 12 circles of the same color were grouped into a triangle or an arrow. All sizes and distances were the same as in the previous condition. In the connected triangle/arrow condition (Figure 1D), colored lines $\left(.48^{\circ}\right.$ in width) formed a connected triangle or an arrow.

Design. Background conditions were administered between subjects, and background similarity and target similarity were administered within subjects. All the combinations of background similarity and target similarity were randomized within blocks, with each combination occurring on an equal number of trials. There were 160 experimental trials, preceded by 16 practice trials, in each background condition.

Procedure. Each trial started with a central fixation dot that appeared for $250 \mathrm{msec}$. After a 250 -msec interval, the first display appeared for $200 \mathrm{msec}$ followed by a 150 - $\mathrm{msec}$ interval, and then the second display appeared for $200 \mathrm{msec}$. At this point, the subjects had to decide, as rapidly and as accurately as possible, whether the two successive central squares were the same or different by pressing one of two response keys. Reaction time (RT) was measured from the appearance of the second display until a response was made. Feedback 
Same Background

Organization

Display 1
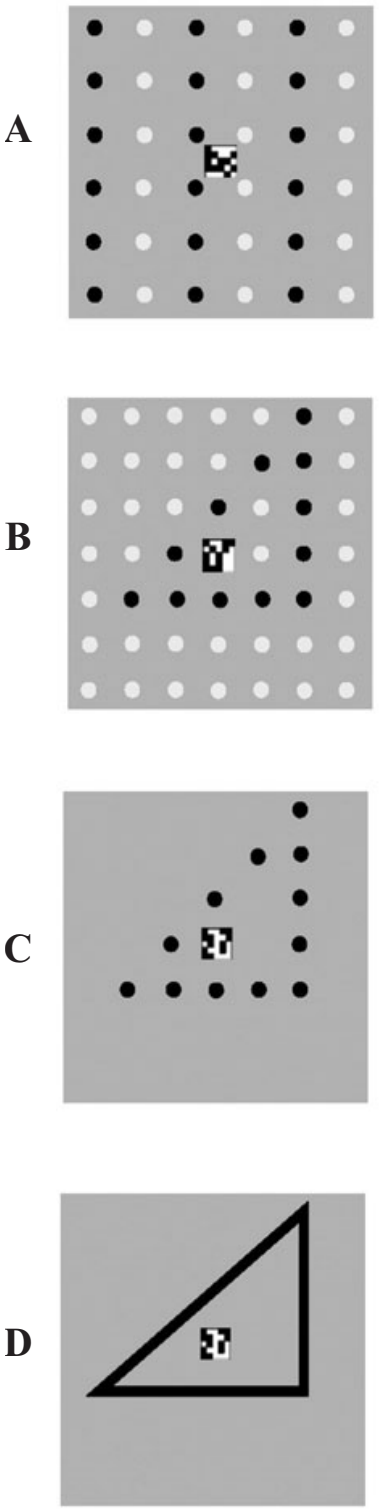

Display 2
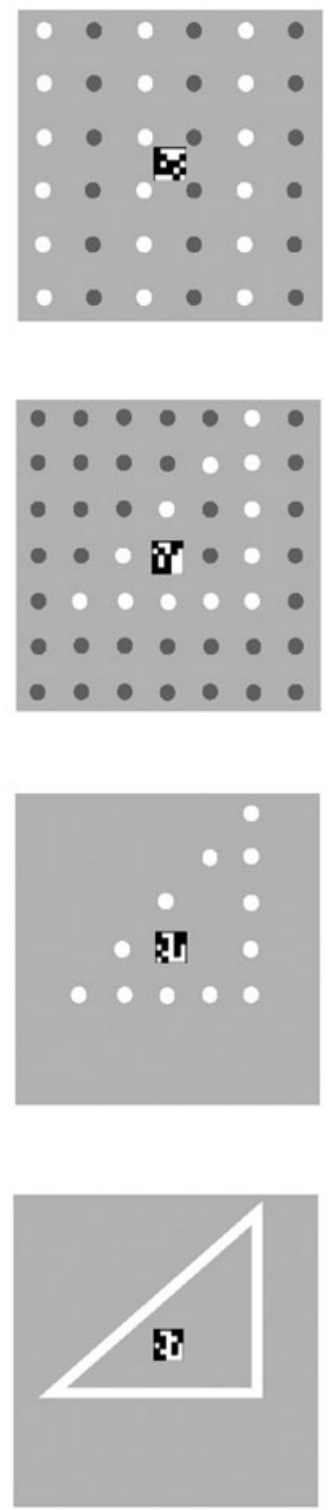

\section{Different Background Organization}

Display 2
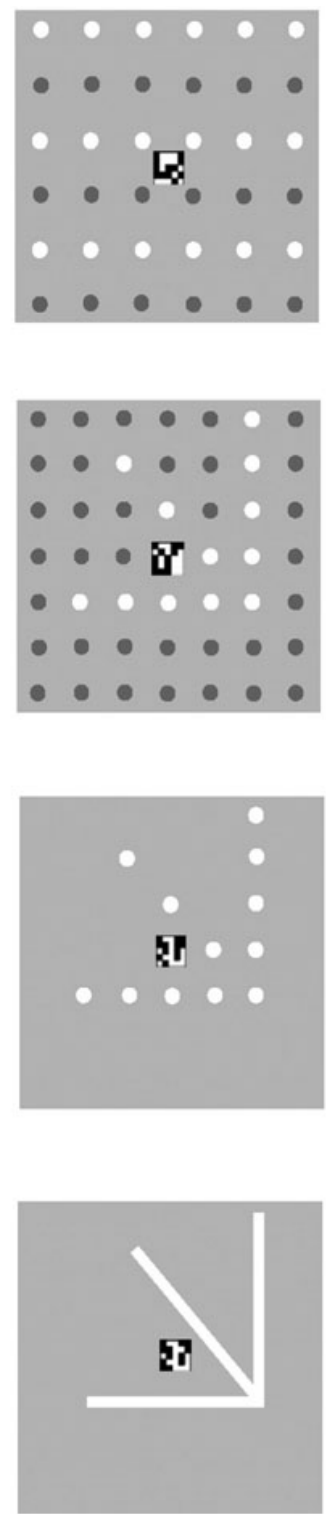

Figure 1. Examples of the stimulus displays in Experiment 1. Two successive displays are presented on each trial. The central target squares in Displays 1 and 2 are either the same or different. The surrounding colored elements are grouped into (A) columns/rows by color similarity, (B) a triangle/arrow by color similarity, (C) a triangle/arrow, or (D) a connected triangle/arrow. This background organization either stays the same across Displays 1 and 2 or changes (independently of whether the central target square changes or not). The colors of the background elements (blue, yellow, red, or green) always change between Displays 1 and 2 - for example, from blue and yellow in Display 1 to red and green in Display 2. In the illustration, black corresponds to blue, light gray to yellow, white to red, and dark gray to green. All colors were equiluminant in the experiment.

about an incorrect response was provided by an auditory tone as soon as the subject responded. The intertrial interval was 1,000 msec.

After subjects responded to the last experimental trial, they were asked the following three open questions about the immediately preceding display: "Did you see anything else besides the central square?"; "What was the pattern in the background?"; and "Which colors appeared in the background?" Subjects who responded negatively to the first question were nevertheless encouraged to answer the other two questions. Finally, the subjects' color vision was tested with the Dvorine Pseudo-Isochromatic Plates test. 


\section{Results and Discussion}

All RT summaries and analyses are based on subjects' median RTs for correct responses. Mean correct RTs and error rates (ERs) are plotted in Figure 2 as a function of target similarity and background similarity for the four background conditions.

The RT and ER data were subjected to a three-factor analysis of variance (ANOVA) that treated background condition as a between-subjects factor and target similarity and background similarity as within-subjects factors. The overall level of target judgments was the same in all background conditions [RT, $F_{\mathrm{S}}<1$; ER, $F(3,52)=$ $\left.1.79, M S_{\mathrm{e}}=138, p>.16\right]$. However, the significant interaction of target similarity, background similarity, and background condition [RT, $F(3,52)=8.14, M S_{\mathrm{e}}=$ $396, p<.0002$; ER, $\left.F(3,52)=4.44, M S_{\mathrm{e}}=35, p<.008\right]$ indicates that the effect of the background on subjects' performance varied as a function of background grouping. Planned specific comparisons were carried out to reveal the effects of the background on target judgments for each condition.

Columns/rows by color similarity (Figure 2A). Target similarity interacted with background similarity for RTs $\left[F(1,13)=16.74, M S_{\mathrm{e}}=288, p<.002\right]$, indicating an influence of the background on subjects' speed of performance. Target-same judgments were faster (by $23 \mathrm{msec}$ ) when the background stayed the same than when it changed $\left[F(1,13)=12.03, M S_{\mathrm{e}}=322, p<.005\right]$, and target-different judgments were faster (by $14 \mathrm{msec}$ ) when the background organization changed than when it stayed the same $\left[F(1,13)=5.93, M S_{\mathrm{e}}=217, p<.03\right]$. No significant effects of background were observed for the accuracy data $(F<1)$.

Triangle/arrow by color similarity (Figure 2B). No significant interaction between target similarity and background similarity was found [RT, $F<1$; ER, $F(1,13)=$ $\left.2.74, M S_{\mathrm{e}}=14, p>.12\right]$. There was no effect of background on the speed and accuracy of target-same judgments $(F \mathrm{~S}<1)$, nor on target-different judgments [RT, $F<1$; ER, $\left.F(1,13)=3.35, M S_{\mathrm{e}}=20, p>.1\right]$.

Triangle/arrow (Figure 2C). The interaction between target similarity and background similarity was not significant [RT, $F<1$; ER, $\left.F(1,13)=3.04, M S_{\mathrm{e}}=27, p>.11\right]$. The background had no influence on the speed and accuracy of target-same judgments $\left(F_{\mathrm{S}}<1\right)$. A closer inspection of the data revealed some influence of the background on target-different judgments, which were more accurate (by $5.3 \%$ ) when the background changed $[F(1,13)=$ $\left.6.34, M S_{\mathrm{e}}=32, p<.03\right]$. Also, target-different judgments tended to be faster (by $9 \mathrm{msec}$ ) when the background changed, but the effect was not significant $[F(1,13)=$ 2.33, $\left.M S_{\mathrm{e}}=276, p>.15\right]$.

Connected triangle/arrow (Figure 2D). Target similarity interacted significantly with background similarity [RT, $F(1,13)=32.95, M S_{\mathrm{e}}=517, p<.0001 ; \mathrm{ER}, F(1,13)=$ $\left.10.4, M S_{\mathrm{e}}=51, p<.007\right]$. Target-same judgments were faster [by $46 \mathrm{msec}, F(1,13)=19.23, M S_{\mathrm{e}}=790, p<$ $.0007]$ and more accurate [by $7.5 \%, F(1,13)=9.87$,
$\left.M S_{\mathrm{e}}=40, p<.008\right]$ when the background stayed the same, and target-different judgments were faster [by $\left.23 \mathrm{msec}, F(1,13)=7.23, M S_{\mathrm{e}}=522, p<.02\right]$ when the background changed. Target-different judgments tended to be more accurate (by $4.8 \%$ ) when the background changed, but the difference was not significant $[F(1,13)=$ $\left.3.12, M S_{\mathrm{e}}=52, p>.10\right]$.

Percentages of correct responses to the surprise question for each background condition are presented in Table 1. Although most subjects in all conditions reported seeing something else besides the central target and correctly reported the color of the background elements, none of the subjects in the columns/rows by color similarity condition and in the triangle/arrow by color similarity condition, and only one $(7 \%)^{1}$ in the triangle/ arrow condition, correctly reported the background organization. On the other hand, 13 of the 14 subjects in the connected triangle/arrow condition correctly reported the background organization.

The finding that connected shapes were available to subjects' awareness converges with Mack et al.'s finding (1992) that subjects were aware of connected columns or rows under conditions of inattention. These findings suggest that connectedness may be critical for conscious perception of unattended shape. Alternatively, these findings might have been due to a greater perceptual salience of the connected shapes, but we rule out this possibility in Experiment 2.

The results for the triangle/arrow by color similarity condition were significantly different from those for the columns/rows condition $\left[F(1,26)=5.7, M S_{\mathrm{e}}=331, p<\right.$ $.03]$, indicating an influence of the background on target judgments for the latter but not for the former. Since both conditions involved the same grouping factor of color similarity, the difference in the results might be attributable to a difference in the complexity of shape formationsimpler for the columns/rows than for the triangle/arrow. Alternatively, the difference might have been due to the need to resolve figure-ground relations for the segregated units in the triangle/arrow by color similarity condition (e.g., designating the group of red elements as a figure and the group of green elements as ground). No such operation was required for the columns/rows condition because all segregated units were designated as "figures" (e.g., red and green columns), contributing to the global orientation of the pattern (horizontal vs. vertical). In the next experiment, we examined further the attentional demands of grouping into a shape and these alternative accounts.

\section{EXPERIMENT 2}

Background elements were arranged into a square/cross by color similarity (Figure 3A), a square/cross with no other background elements (Figure 3B), and a vertical/ horizontal line by color similarity (Figure 3C). Shape formation for the latter was as simple as for the columns/ rows condition in Experiment 1 (requiring only determi- 


\section{A. Columns/Rows by Color Similarity}
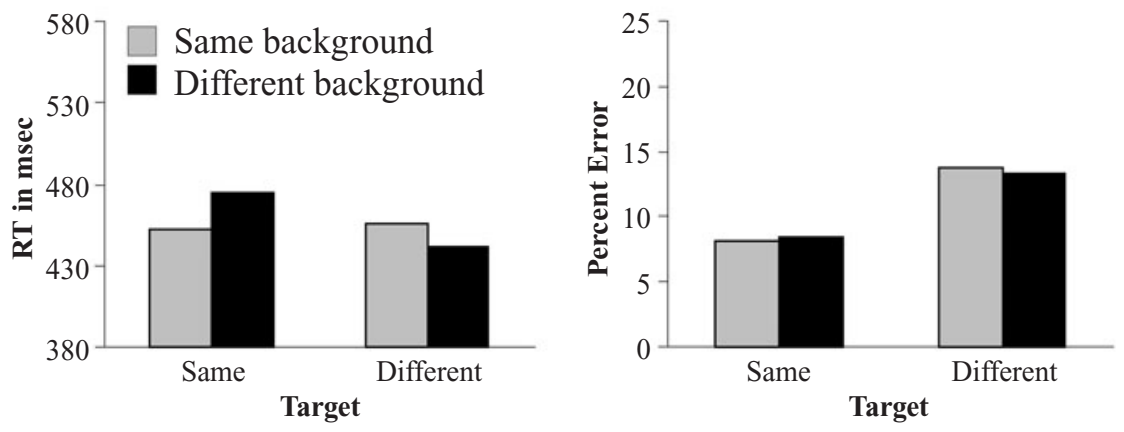

B. Triangle/Arrow by Color Similarity
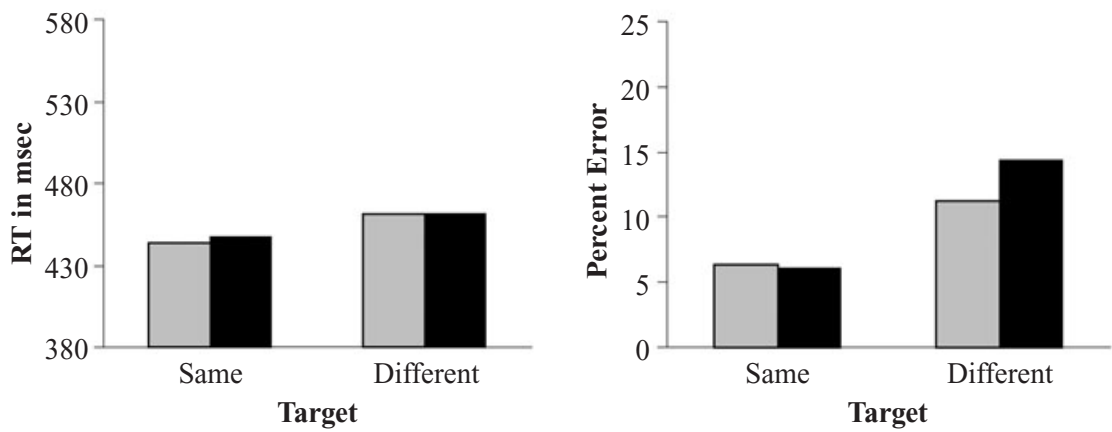

\section{Triangle/Arrow}
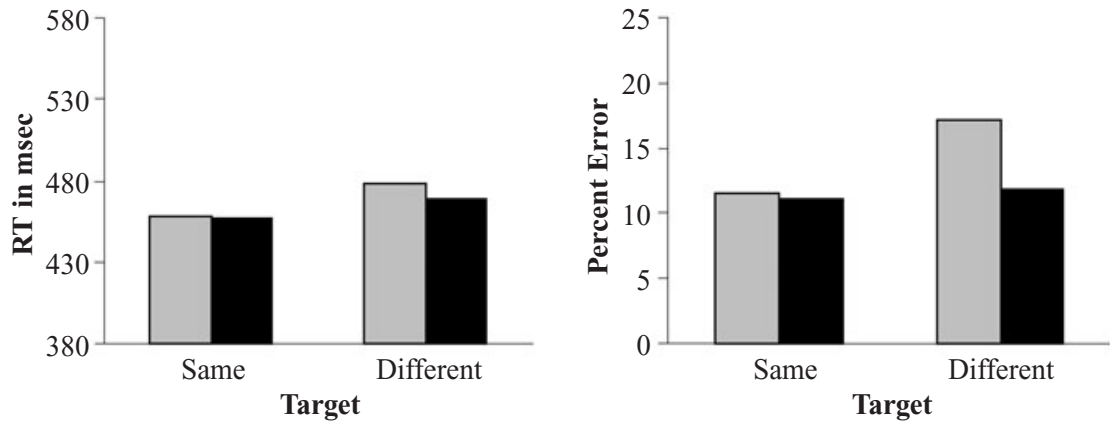

D. Connected Triangle/Arrow
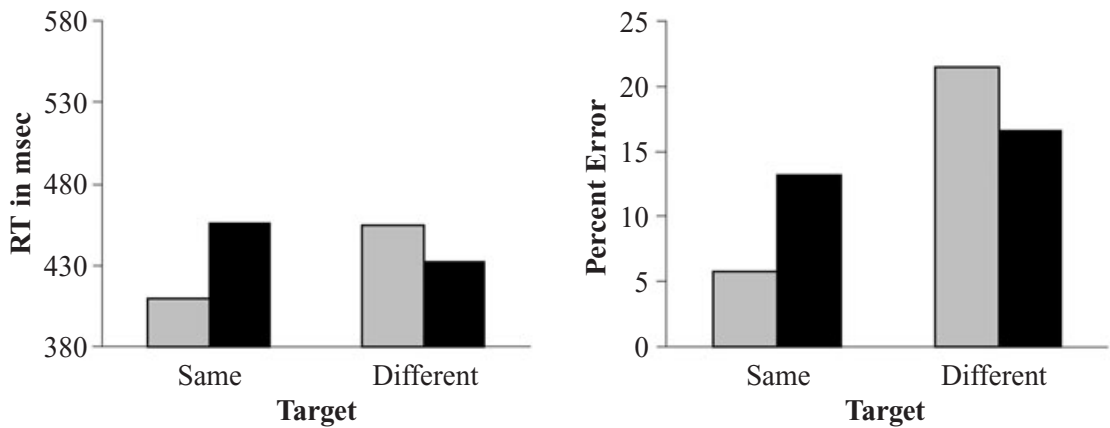

Figure 2. Mean correct RTs (left panel) and error rates (right panel) for target-same and target-different judgments as a function of background similarity (same or different) for each background condition in Experiment 1. 
Table 1

Percentage of Correct Responses to Surprise Questions for the Four Types of Organization in Experiment 1

\begin{tabular}{lccc}
\hline \multicolumn{1}{c}{ Type of Organization } & Something & Color & Organization \\
\hline Columns/rows by color similarity & $93 \%$ & $86 \%$ & $0 \%$ \\
Triangle/arrow by color similarity & $100 \%$ & $93 \%$ & $0 \%$ \\
Triangle/arrow & $79 \%$ & $71 \%$ & $7 \%$ \\
Connected triangle/arrow & $93 \%$ & $93 \%$ & $93 \%$ \\
\hline
\end{tabular}

nation of the orientation of the grouped elements), but unlike the columns/rows condition, it also required resolving figure-ground relations, as in the square/cross by color similarity condition. The question was whether attentional demands would vary as a function of the complexity of shape formation, or rather as a function of the need to resolve figure-ground relations. In the fourth condition, line segments were grouped into a disconnected square/cross (Figure 3D) with salience similar to that for the connected shape in Experiment 1. We employed this condition in order to examine whether subjects' awareness of the connected shape was due to connectedness or to perceptual salience.

\section{Method}

Subjects. Forty-eight students at the University of Haifa served as subjects (12 in each of the four background conditions). None had participated in Experiment 1.

Stimuli. The central target square was the same as in Experiment 1 . In the square/cross by color similarity condition (Figure 3A), 48 circles, 8 of one color and 40 of a different color, were grouped by color similarity into a square or a cross (with each side subtending $3.36^{\circ}$ ). All measurements of the background circles, including the distance of the most central circles from the target, were identical to those of the triangle/arrow by color similarity in Experiment 1 . In the square/cross condition (Figure 3B), eight circles, all of the same color, were grouped into a square or a cross (of the same sizes and distances as in the previous condition). In the vertical/ horizontal line by color similarity condition (Figure 3C), 36 circles, 6 of one color and 30 of a different color, were grouped into a vertical or a horizontal line by color similarity. All measurements of the background circles, including the distance of the most central circles from the target, were identical to those of the columns/rows in Experiment 1. In the disconnected square/cross condition (Figure $3 \mathrm{D}$ ), line segments, $2.4^{\circ}$ each, were grouped into a square or a cross.

\section{Results and Discussion}

Mean correct RTs and ERs are plotted in Figure 4 as a function of target similarity and background similarity for the four background conditions. The ANOVA performed on the RT and ER data showed a significant three-way interaction of target similarity, background similarity, and background condition [RT, $F(3,44)=5.14, M S_{\mathrm{e}}=950$, $p<.004$; ER, $\left.F(3,44)=15.7, M S_{\mathrm{e}}=21, p<.0001\right]$. There was no significant difference in the overall level of performance among the background conditions [RT, $F<$ 1; ER, $\left.F(3,44)=2.14, M S_{\mathrm{e}}=110, p>.11\right]$.

Square/cross by color similarity (Figure 4A). Target similarity did not interact significantly with background similarity [RT, $F<1$; ER, $F(1,11)=1.82, M S_{\mathrm{e}}=23$, $p>$.2]. There was no effect of background on the speed and accuracy of target-same judgments $(F \mathbf{s}<1)$, nor on target-different judgments [RT, $F<1$; ER, $F(1,11)=$ $\left.3.0, M S_{\mathrm{e}}=17, p>.11\right]$.

Square/cross (Figure 4B). Target similarity interacted significantly with background similarity [RT, $F(1,11)=$ 6.27, $M S_{\mathrm{e}}=512, p<.03$; ER, $F(1,11)=25.06, M S_{\mathrm{e}}=$ $18, p<.0004$ ]. Target-same judgments were faster [by $\left.30 \mathrm{msec} ; F(1,11)=5.63, M S_{\mathrm{e}}=899, p<.04\right]$ and more accurate [by $7 \% ; F(1,11)=26.71, M S_{\mathrm{e}}=11, p<.0003$ ] when the background stayed the same, and target-different judgments were more accurate (by 5\%) when the background organization changed $\left[F(1,11)=5.12, M S_{\mathrm{e}}=30\right.$, $p<.05]$. There was no significant effect of the background on the speed of target-different judgments $(F<1)$.

Vertical/horizontal line by color similarity (Figure 4C). Target similarity did not interact significantly with background similarity $\left[F_{\mathrm{S}}<1\right.$, for RT and ER]. There was no effect of background on the speed and accuracy of either target-same judgments or target-different judgments $(F \mathrm{~s}<1)$.

Disconnected square/cross (Figure 4D). Target similarity interacted significantly with background similarity [RT, $F(1,11)=15.54, M S_{\mathrm{e}}=1,264, p<.003$; ER, $F(1,11)=$ 45.08, $\left.M S_{\mathrm{e}}=21, p<.0001\right]$. Target-same judgments were faster [by $69 \mathrm{msec} ; F(1,11)=18.11, M S_{\mathrm{e}}=1,566$, $p<.002$ ] and more accurate [by $12 \% ; F(1,11)=25.07$, $\left.M S_{\mathrm{e}}=33, p<.0004\right]$ when the background stayed the same, and target-different judgments were more accurate (by $6 \%$ ) when the background organization changed $\left[F(1,11)=17.96, M S_{\mathrm{e}}=12, p<.002\right]$. The tendency of target-different judgments to be faster (by $12 \mathrm{msec}$ ) when background organization changed was not significant $\left[F(1,11)=1.17, M S_{\mathrm{e}}=761, p>.3\right]$. The results in this condition did not differ from those in the square/cross condition [RT, $F(1,22)=3.93, M S_{\mathrm{e}}=888, p>.06$; ER, $\left.F(1,22)=2.46, M S_{\mathrm{e}}=19, p>.13\right]$.

Percentages of correct responses to each of the surprise questions are presented in Table 2. Most subjects reported seeing something other than the target square and correctly reported the color of the background elements. None of the subjects in the square/cross by color similarity condition, and only $3(25 \%)$ in each of the square/cross, vertical/horizontal line, and disconnected square/cross conditions correctly reported the background organization. ${ }^{2}$

The results were the same for the vertical/horizontal line by color similarity condition as for the square/cross by color similarity condition (Experiment 2) and the triangle/ arrow by color similarity condition (Experiment 1), indicating no effect of the background on target judgments. This finding suggests that the difference in results between the columns/rows condition (an effect of the background) and the shape by color similarity conditions (no effect of the background) is not likely to have been due to a difference in the complexity of shape formation per se. Rather, these results support the hypothesis that grouping into a shape by color similarity demanded attention because it 


\section{Same Background \\ Organization}

Display 1

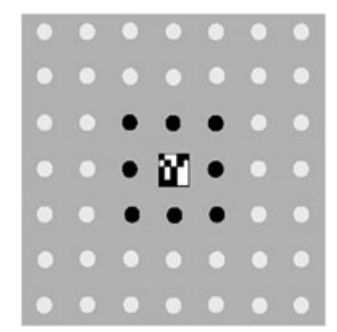

Display 2
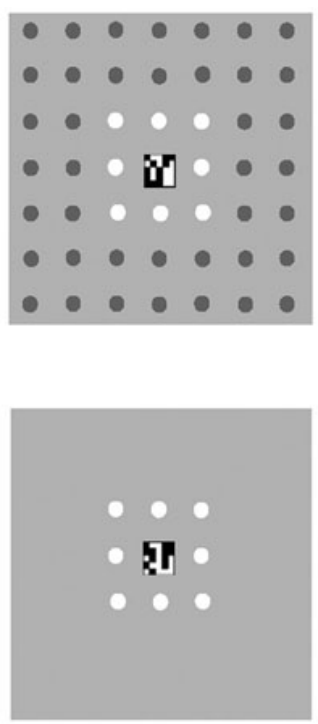

B

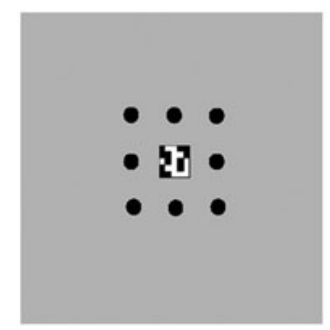

C

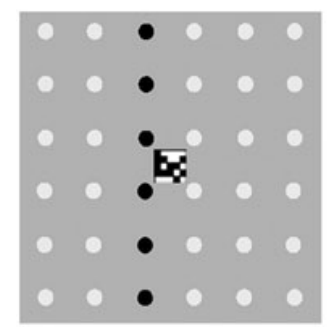

D

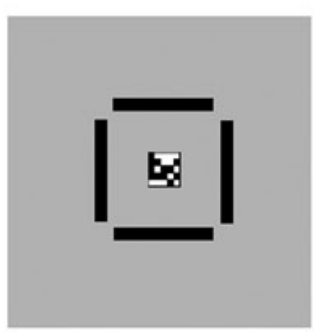

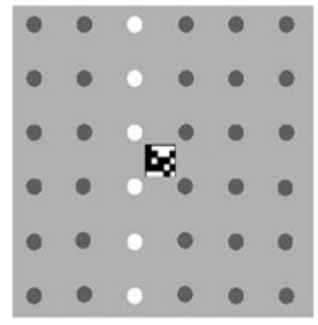

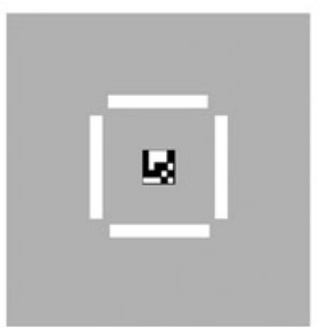

\section{Different Background \\ Organization}

Display 2
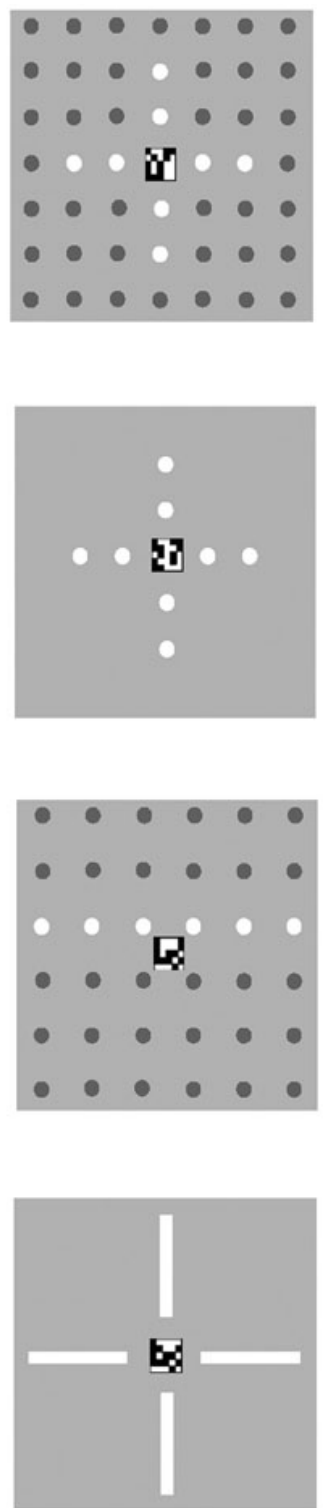

Figure 3. Examples of the stimulus displays in Experiment 2. The central target squares (made up of black and white pixels) in Displays 1 and 2 are either the same or different. The surrounding colored elements are organized into (A) a square/cross by color similarity, (B) a square/cross, (C) a vertical/horizontal line by color similarity, or (D) line segments grouped into a square/cross. This background organization stays the same across Displays 1 and 2 or changes (independently of whether the target square changes or not). The colors of the background elements (blue, yellow, red, or green) always change between Displays 1 and 2-for example, from blue and yellow in Display 1 to red and green in Display 2. In the illustration, black corresponds to blue, light gray to yellow, white to red, and dark gray to green. All colors were equiluminant in the experiment.

required resolving figure-ground relations for the segregated units, whereas no such operation was required in the columns/rows condition.

Note that the difference in results between the columns/ rows condition and the shape by color similarity condi- tions cannot be explained away as an artifact of the distance of the most central background elements from the central target nor of the amount of actual physical change (i.e., number of elements changing between successive displays). One could argue that the background 


\section{A. Square/Cross by Color Similarity}
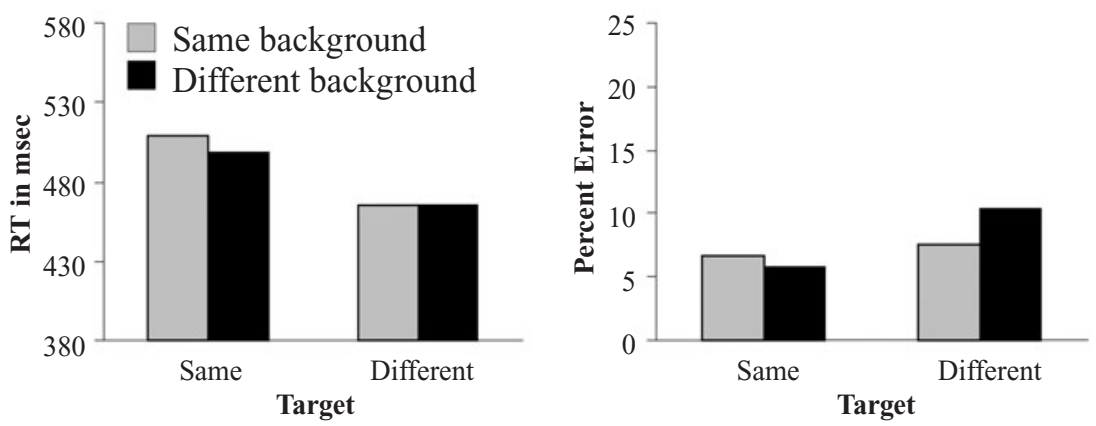

\section{B. Square/Cross}
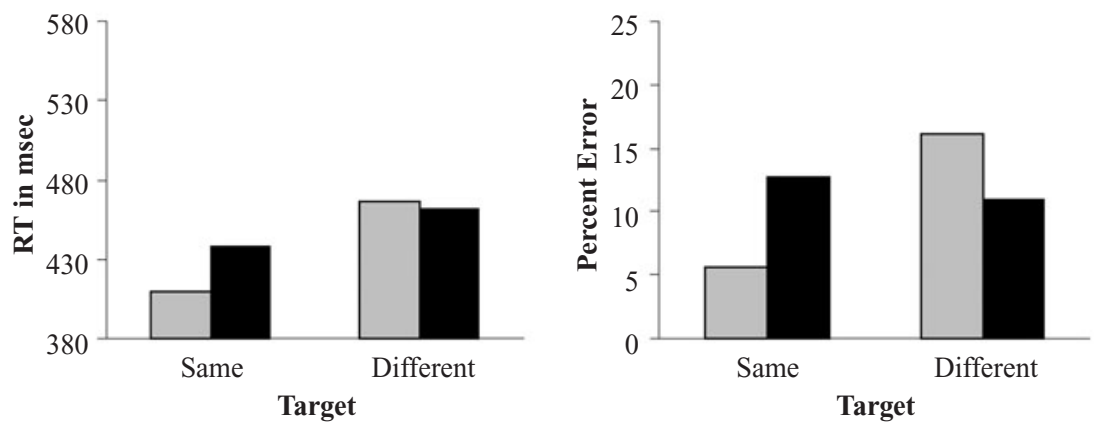

C. Vertical/Horizontal Line by Color Similarity
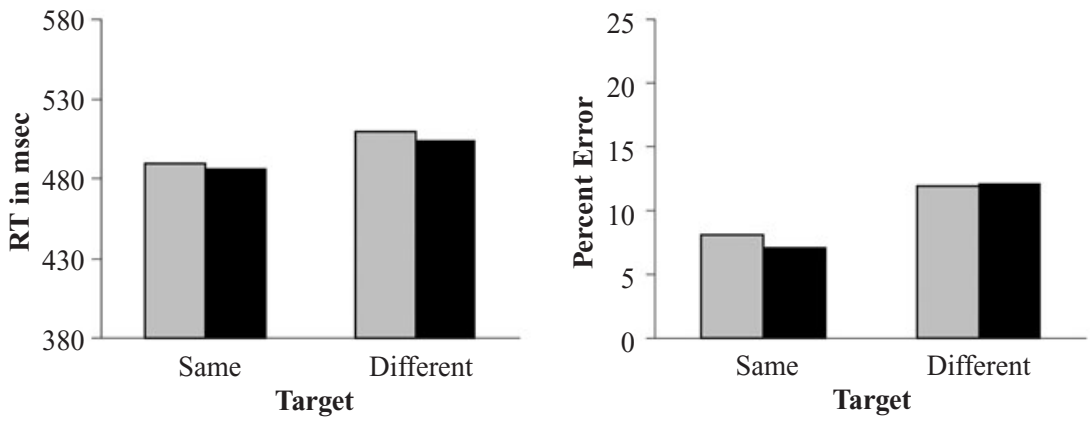

\section{Disconnected Square/Cross}
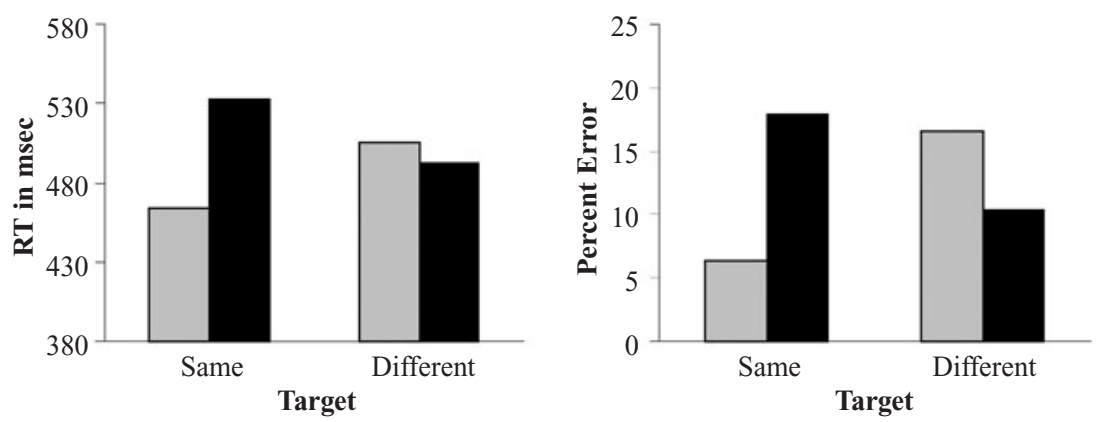

Figure 4. Mean correct RTs (left panel) and error rates (right panel) for target-same and target-different judgments as a function of background similarity (same or different) for each background condition in Experiment 2. 
Table 2

Percentage of Correct Responses to Surprise Questions for the Four

Types of Organization in Experiment 2

\begin{tabular}{lccc}
\hline \multicolumn{1}{c}{ Type of Organization } & Something & Color & Organization \\
\hline Square/cross by color similarity & $100 \%$ & $92 \%$ & $0 \%$ \\
Square/cross & $100 \%$ & $92 \%$ & $25 \%$ \\
Vertical/horizontal line by color similarity & $92 \%$ & $83 \%$ & $25 \%$ \\
Disconnected square/cross & $92 \%$ & $83 \%$ & $25 \%$ \\
\hline
\end{tabular}

effect observed in the columns/rows condition was due to implicit processing of the most central background elements that might have fallen in the attended area because of their close proximity to the target, rather than to grouping of the entire background display. However, although the most central elements were indeed closer to the target in the columns/rows condition than in the triangle/arrow and the square/cross conditions, they were exactly as close in the vertical/horizontal line condition. Therefore, the finding of no background effect for the vertical/horizontal line condition rules out this account. Alternatively, the background effect in the columns/rows condition might be accounted for by the greater amount of physical change in this condition than in the shape by color similarity conditions. This account, however, cannot easily explain the finding of no background effect for the square/cross, the vertical/horizontal line, and the triangle/arrow conditions, although the amount of physical change was larger for the two former conditions than for the latter one.

The results for the square/cross condition with no other background elements imply background grouping, suggesting that grouping into a shape can occur without attention when segregation is relatively easy. Alternatively, these results could be due to unique onsets and offsets of the background that might have attracted attention. However, the presence of background grouping in the absence of unique onsets and offsets (i.e., in the columns/rows condition where all background circles were present all the time) is incompatible with this interpretation.

Contrary to the results for the square/cross condition, only a small effect of the background was found for the comparable triangle/arrow condition (Experiment 1). This finding suggests that the degree of shape "goodness" imposes differential attentional demands. It has been proposed that pattern "goodness" is directly linked to the number of symmetries in the pattern (Feldman, 2000; Garner, 1974; Palmer, 1991), implying that squares and crosses are "better" shapes than isosceles triangles and arrows.

\section{GENERAL DISCUSSION}

The results of these experiments show that, in agreement with previous findings (Driver et al., 2001; Mack et al., 1992; Moore \& Egeth, 1997), subjects are unable to report the grouping of elements under conditions of inat- tention. Only when the unattended background consisted of a connected shape were subjects aware of it. However, the pattern of RT and ER of the same-different judgments implies that background elements were grouped into columns or rows by color similarity, and into a square or a cross, and to some extent into a triangle or an arrow, when no other background elements were present. In contrast, there was no indication that background elements were grouped by color similarity into a triangle/arrow, a square/cross, or a horizontal/vertical line.

These results indicate that both clustering and shape formation can take place without attention and thus are incompatible with the view of a dichotomy between these processes in terms of attentional demands, or with the claim that connectedness is critical for preattentive shape recovery (Trick \& Enns, 1997).

Rather, our results suggest that a continuum of attentional demands exists as a function of the processes involved in grouping and the conditions prevailing for each process. Grouping into a shape can occur under inattention when no segregation from other elements is involved, depending on shape "goodness" or simplicity of shape formation. Grouping that involves segregation from other elements can also take place without attention when all segregated units are designated as "figures," as in grouping into columns/rows by color similarity. Resolving figure-ground relations between segregated units, however, appears to demand attention. When such an operation was required, no background grouping was observed, regardless of the simplicity of shape formation (i.e., vertical/horizontal line vs. square/ cross and triangle/ arrow) or the relative "goodness" of the shape (i.e., square/ cross vs. triangle/arrow). Note that although the requirement to resolve figure-ground relations suffices to impose attentional demands, it need not be necessary. It is possible, for example, that grouping into columns/rows would have demanded attention if it were based on a certain kind of shape similarity instead of color similarity (e.g., arrows vs. crosses; see Han \& Humphreys, 1999).

A concern for any attempt to examine processing without attention is whether or not conditions of inattention are indeed employed. Our conditions seem to fit Mack et al.'s (1992) definition of inattention. The target judgment task was sufficiently demanding to absorb attention, presumably leaving no spare attention for background processing, and background grouping was irrelevant to the target task. We have also noted that the results cannot have been due to implicit processing of the most central 
background elements that might have fallen in the attended area, nor to capturing of attention by the background in some conditions. Furthermore, the finding that subjects maintained the same overall level of performance in target judgments across all conditions strongly suggests that the differential influence of the background on target judgments in different conditions was indeed indicative of the differential attentional requirements of the groupings.

Regardless of attentional demands, our results show that the products of grouping are not available to awareness without attention. Rather, connectedness may be critical for conscious perception of unattended shape.

In a final note, it may be appropriate to consider an alternative approach to the one we have taken here - namely, that attention is a unitary resource, and that grouping processes vary in their requirements of it. A more dynamic view of the visual system has been recently proposed (e.g., Di Lollo, Kawahara, Zuvic, \& Visser, 2001), according to which the efficiency of early perceptual processing depends on whether the system is optimally configured to handle the task at hand. This view implies that results pertaining to attentional demands of certain processes may depend not only on the nature of these processes but also on the current configuration of the system. Although this proposal does not detract from our findings and conclusion that grouping is not a unitary process but rather a multiplicity of processes, it suggests that the results are no less a function of the attentional processes involved in the target task. Therefore, different background groupings may be accomplished with a different primary task, depending on how they fit the current configuration. Yet, it may well be the case that our results reveal some constraints on the complexity to which the visual system involved in grouping can be configured. This is an issue worth pursuing. In any case, our findings clearly show that the relation between grouping and attention is not unidimensional.

\section{REFERENCES}

Behrmann, M., \& Kimchi, R. (2003). What does visual agnosia tell us about perceptual organization and its relationship to object perception? Journal of Experimental Psychology: Human Perception \& Performance, 29, 19-42.

BEN-Av, M. B., SAGI, D., \& BrAUN, J. (1992). Visual attention and perceptual grouping. Perception \& Psychophysics, 52, 277-294.

Di Lollo, V., Kawahara, J., Zuvic, S. M., \& VisSer, T. A. W. (2001). The preattentive emperor has no clothes: A dynamic redressing. Journal of Experimental Psychology: General, 130, 479-492.

Driver, J., Davis, G., Russell, C., Turatto, M., \& Freeman, E. (2001). Segmentation, attention and phenomenal visual objects. $\underline{\text { Cog- }}$ nition, 80, 61-95.

ENNS, J. T., \& KInGSTONE, A. (1995). Access to global and local properties in visual search for compound stimuli. Psychological Science, $\underline{\text { 6, 283-291. }}$
Feldman, J. (2000). Bias toward regular form in mental shape spaces. Journal of Experimental Psychology: Human Perception \& Performance, 26, 152-165.

GARNER, $\overline{\mathrm{W}} . \overline{\mathrm{R} .}$ (1974). The processing of information and structure. Hillsdale, NJ: Erlbaum.

HaN, S., \& Humphreys, G. W. (1999). Interactions between perceptual organization based on Gestalt laws and those based on hierarchical processing. Perception \& Psychophysics, 61, 1287-1298.

Han, S., HumpHreYs, G. W., \& CHEN, L. (1999). Parallel and competitive processes in hierarchical analysis: Perceptual grouping and encoding of closure. Journal of Experimental Psychology: Human Perception \& Performance, 25, 1411-1432.

JuLESZ, B. (1984). A brief outline of the texton theory of human vision. Trends in Neurosciences, 7, 41-45.

KIMCHI, R. (1998). Uniform connectedness and grouping in the perceptual organization of hierarchical patterns. Journal of Experimental Psychology: Human Perception \& Performance, 24, 1105-1118.

KoffKa, K. (1935). Principles of Gestalt psychology. New York: Harcourt, Brace \& World.

Mack, A., Tang, B., Tuma, R., Kahn, S., \& Rock, I. (1992). Perceptual organization and attention. Cognitive Psychology, 24, 475-501.

MARR, D. (1982). Vision: A computational investigation into the human representation and processing of visual information. San Francisco: Freeman.

Moore, C. M., \& Egeth, H. (1997). Perception without attention: Evidence of grouping under conditions of inattention. Journal of Experimental Psychology: Human Perception \& Performance, 23, 339352.

NeIsSER, U. (1967). Cognitive psychology. New York: Appleton-CenturyCrofts.

Palmer, S. E. (1991). Goodness, Gestalt, groups, and Garner: Local symmetry subgroups as a theory of figural goodness. In G. R. Lockhead $\&$ J. R. Pomerantz (Eds.), The perception of structure: Essays in honor of Wendell R. Garner (pp. 23-39). Washington, DC: American Psychological Association.

Rock, I. (1986). The description and analysis of object and event perception. In K. R. Boff, L. Kaufman, \& J. P. Thomas (Eds.), Handbook of perception and human performance (Vol. 2, pp. 33.1-33.71). New York: Wiley.

Russell, C., Howlett, H., \& Driver, J. (2000, April). Implicit measures of visual grouping under conditions of inattention. Paper presented at the Seventh Annual Meeting of the Cognitive Neuroscience Society, San Francisco.

Treisman, A. (1982). Perceptual grouping and attention in visual search for features and for objects. Journal of Experimental Psychology: Human Perception \& Performance, 8, 194-214.

TRICK, L. M., \& ENNS, J. T. (1997). Clusters precede shapes in perceptual organization. Psychological Science, $\mathbf{8}, 124-129$.

\section{NOTES}

1. It is not possible to state what chance performance would be since the questions were open. However, percentage of correct responses to the organization question was significantly lower than to the color question $(p<.0001)$.

2. See note 1. Percentage of correct responses to the organization question was significantly lower than to the color question in each of these conditions $(p<.02)$.

(Manuscript received March 27, 2003; revision accepted for publication August 20, 2003.) 